Harvey Rosen, Department of Mathematics, University of Alabama,

Tuscaloosa, AL. 35487 e-mail: Hrosen@gp.as.ua.edu

\title{
POROUS AND BOUNDARY SETS IN DARBOUX-LIKE FUNCTION SPACES
}

\begin{abstract}
We show which subspaces of Darboux-like real function spaces are porous or boundary sets with the metric of uniform convergence.
\end{abstract}

We are interested in the size of one function subspace inside another function space. Porosity is one such measure of this. In a metric space $(X, d)$, $B(x, r)$ denotes the open ball centered at $\mathrm{x}$ with radius $r>0$. For $M \subset X$, $x \in X$, and $r>0$, we let $\gamma(x, r, M)$ denote the supremum of the set of all $s>0$ for which there exists $z \in X$ such that $B(z, s) \subset B(x, r) \backslash M$. $M$ is porous at $x$ if $p(x, M)=\lim \sup _{r \rightarrow 0+} \frac{\gamma(x, r, M)}{r}>0 . M$ is porous in $\mathrm{X}$ if $M$ is porous at each $x \in \bar{M}$. (Others define porosity for only $x \in M$.) For example, in $X=\mathbb{R}$, $M_{1}=\left\{ \pm \frac{1}{2^{n}}: n=1,2,3, \ldots\right\}$ is porous at 0 because $p\left(0, M_{1}\right)=\frac{1}{4}$, but $M_{2}=\left\{ \pm \frac{1}{n}: n=1,2,3, \ldots\right\}$ is not porous at 0 because $p\left(0, M_{2}\right)=0$. A porous set $M$ has to be a boundary set in $X$; i.e., $\overline{X \backslash M}=X$. In this paper, we determine the porosity of subspaces of Darboux-like function spaces. The following functions $f: \mathbb{R} \rightarrow \mathbb{R}$ belong to these abbreviated classes of functions. Historically, for Baire class 1 functions $f: \mathbb{R} \rightarrow \mathbb{R}$, these classes (except for $C$, $W C I V P$, and $P B$ ) become equal.

1. $C$ - the class of continuous functions.

2. $P C-f: \mathbb{R} \rightarrow \mathbb{R}$ is a peripherally continuous function if for each $x \in \mathbb{R}$ and for all open neighborhoods $U$ of $x$ and $V$ of $f(x)$, there exists an open neighborhood $W$ of $x$ such that $W \subset U$ and $f(\operatorname{bd}(W)) \subset V$.

Key Words: porous sets, boundary sets, spaces of Darboux-like real functions

Mathematical Reviews subject classification: 26A15, 54C35

Received by the editors March 9, 2000 
3. $D$ - $f$ is a Darboux function if $f(J)$ is connected for each connected set $J \subset \mathbb{R}$.

4. Conn - $f$ is a connectivity function if the graph of $f$ restricted to $J$ is a connected subset of $\mathbb{R}^{2}$ for each connected set $J \subset \mathbb{R}$.

5. $A C-f: \mathbb{R} \rightarrow \mathbb{R}$ is almost continuous if each open neighborhood in $\mathbb{R}^{2}$ of the graph of $f$ contains the graph of a continuous function $g: \mathbb{R} \rightarrow \mathbb{R}$.

6. Ext - $f$ is extendable if there is a function $F: \mathbb{R}^{2} \rightarrow \mathbb{R}$ such that $F(x, 0)=f(x)$ for all $x \in \mathbb{R}$ and the graph of the restriction $F\lceil J$ is connected for each connected set $J \subset \mathbb{R}^{2}$.

7. $P R-f$ has a perfect road if for each $x \in \mathbb{R}$ there exists a perfect set $P$ having $x$ as a bilateral limit point such that $f \uparrow P$ is continuous at $x$. We refer to $P$ as a perfect road at $x$.

8. WCIVP - $f$ has the weak Cantor intermediate value property if for each $x<y$ with $f(x) \neq f(y)$ there is a Cantor set $C \subset(x, y)$ such that $f(C)$ lies between $f(x)$ and $f(y)$.

9. $C I V P$ - $f$ has the Cantor intermediate value property if for each $x<y$ with $f(x) \neq f(y)$ and for each Cantor set $K$ between $f(x)$ and $f(y)$ there is a Cantor set $C \subset(x, y)$ such that $f(C) \subset K$.

10. $S C I V P$ - $f$ has the strong Cantor intermediate value property if in the preceding definition $C$ can be chosen so that $f \uparrow C$ is also continuous.

11. $P B$ - $f$ has property B if for each pair $I, J$ of open intervals $I \cap f^{-1}(J)$ whenever uncountable contains a nonempty perfect set.

12. $Z c-f$ satisfies Zahorski's condition if for each $a$, each set $\{x: f(x)<a\}$ and $\{x: f(x)>a\}$ whenever nonempty is bilaterally $\mathfrak{c}$-dense in itself.

13. $Z \omega-f$ satisfies condition $Z \omega$ if for each $a$, each set $\{x: f(x)<a\}$ and $\{x: f(x)>a\}$ whenever nonempty is bilaterally dense in itself.

These definitions could have been given instead for classes of functions $f$ : $[0,1] \rightarrow \mathbb{R}$. Each function space has on it the metric $d$ of uniform convergence defined by $d(f, g)=\min \{1, \sup \{|f(x)-g(x)|: x \in \mathbb{R}\}\}$, and unless otherwise specified, the closure of a function space is taken in the class $\mathbb{R}^{\mathbb{R}}$ of all functions from $\mathbb{R}$ into $\mathbb{R}$.

The following chart, in which $\rightarrow$ indicates proper inclusion, was lifted from [10] and [6]. In [13], we determine which spaces are porous or boundary sets 
for the chain of spaces of functions $f: \mathbb{R} \rightarrow \mathbb{R}$ in the top row of the chart. We indicate in the rest of the chart which function spaces are determined in this paper to be porous or boundary sets.

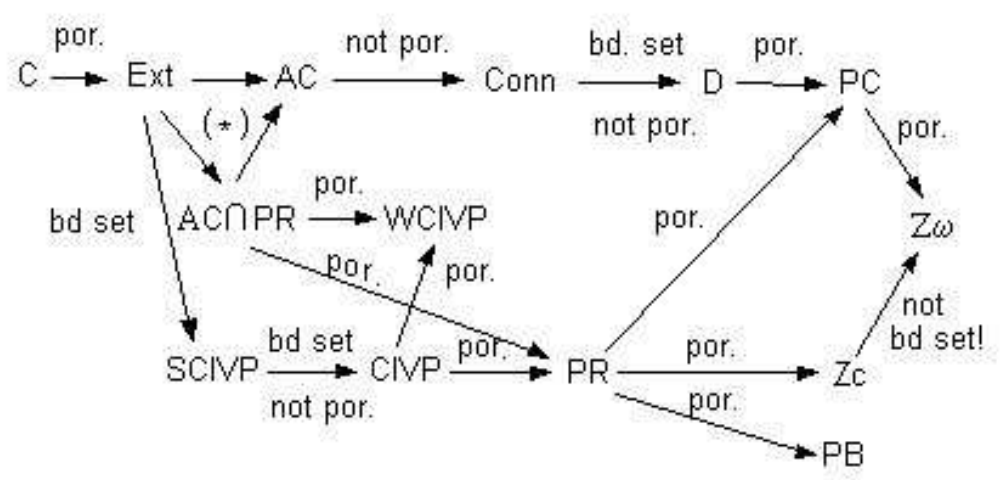

Whether or not Ext is porous in SCIVP and what the situation is for the commutative diagram $(*)$ are left as open problems.

If $A$ is a subspace of $B$ and $B$ is porous in $C$, then $A$ is porous in $C$. This is also true if "porous" is replaced by "a boundary set." For example, according to the chart, Conn is porous in $P C$ because $D$ is porous in $P C$.

We will invoke the following construction often. Suppose $f: \mathbb{R} \rightarrow \mathbb{R}$ is continuous at some point $x_{0} \in \mathbb{R}$, and suppose $h:[0,1] \rightarrow[0,1]$ is any function. Given $r>0$ there exists a $\delta>0$ such that $f\left[x_{0}-\delta, x_{0}+\delta\right] \subset$ $\left(f\left(x_{0}\right)-\frac{r}{4}, f\left(x_{0}\right)+\frac{r}{4}\right)$. We describe what it means to say that "g is the function obtained from $f$ by gluing a copy of $h$ at $x_{0}$." Let $f_{0}$ be a scaled-down copy of $h$ to the rectangle $\left[x_{0}-\frac{\delta}{2}, x_{0}+\frac{\delta}{2}\right] \times\left[f\left(x_{0}\right)-\frac{r}{4}, f\left(x_{0}\right)+\frac{r}{4}\right]$ instead of $[0,1] \times$ $[0,1]$. Namely, $f_{0}(x)=\frac{r}{2} h\left(\frac{1}{\delta}\left(x-x_{0}+\frac{\delta}{2}\right)\right)+f\left(x_{0}\right)-\frac{r}{4}$. We obtain a function $g: \mathbb{R} \rightarrow \mathbb{R}$ by gluing this copy, $f_{0}$, of $h$ into the rectangle $\left[x_{0}-\frac{\delta}{2}, x_{0}+\frac{\delta}{2}\right] \times$ $\left[f\left(x_{0}\right)-\frac{r}{4}, f\left(x_{0}\right)+\frac{r}{4}\right]$ and connecting it linearly to the rest of the graph of $f$ outside the larger rectangle $\left[x_{0}-\delta, x_{0}+\delta\right] \times\left[f\left(x_{0}\right)-\frac{r}{4}, f\left(x_{0}\right)+\frac{r}{4}\right]$. That 
is,

$$
g(x)= \begin{cases}f(x) & \text { if } x \notin\left(x_{0}-\delta, x_{0}+\delta\right) \\ l_{1}(x) & \text { if } x \in\left[x_{0}-\delta, x_{0}-\frac{\delta}{2}\right] \\ f_{0}(x) & \text { if } x \in\left(x_{0}-\frac{\delta}{2}, x_{0}+\frac{\delta}{2}\right) \\ l_{2}(x) & \text { if } x \in\left[x_{0}+\frac{\delta}{2}, x_{0}+\delta\right],\end{cases}
$$

where $l_{1}$ and $l_{2}$ are linear functions such that $l_{1}\left(x_{0}-\delta\right)=f\left(x_{0}-\delta\right), l_{1}\left(x_{0}-\frac{\delta}{2}\right)=$ $f_{0}\left(x_{0}-\frac{\delta}{2}\right), l_{2}\left(x_{0}+\frac{\delta}{2}\right)=f_{0}\left(x_{0}+\frac{\delta}{2}\right)$, and $l_{2}\left(x_{0}+\delta\right)=f\left(x_{0}+\delta\right)$. Notice that $d(f, g)<\frac{r}{2}$. In the proofs, $g$ will inherit certain properties $h$ has.

Theorem 1. $A C \cap P R$ is porous in $W C I V P$.

Proof. Suppose $f \in \overline{A C \cap P R}$. (This closure taken in $W C I V P$ is the same as the closure taken in $\mathbb{R}^{\mathbb{R}}$.)

Case 1: $f$ is continuous at some $x_{0} \in \mathbb{R}$.

Let $h:[0,1] \rightarrow[0,1]$ be the function defined by

$$
h(x)= \begin{cases}1 & \text { if } x=0,1 \\ \left|x-\frac{1}{2}\right| & \text { if } 0<x<1 .\end{cases}
$$

Notice $h \in W C I V P \backslash P R$. For each $0<r \leq 1$, we can glue in a copy of $h$ at $x_{0}$ to obtain from $f$ a function $g: \mathbb{R} \rightarrow \mathbb{R}$ in $W C I V P \backslash P R$ with $d(f, g)<\frac{r}{2}$. In the space $W C I V P, B\left(g, \frac{r}{8}\right) \subset B(f, r) \backslash P R$. Since $\gamma(f, r, A C \cap P R) \geq \frac{r}{8}$, $p(f, A C \cap P R) \geq \frac{1}{8}>0$ implies $A C \cap P R$ is porous at $f$.

Case 2: $f$ is continuous at no point.

Then since $f \in \overline{A C} \subset \bar{D}, \bar{f} \cap(\{x\} \times \mathbb{R})$ is a nondegenerate interval for every $x \in \mathbb{R}$. It follows from [12], [5] that the graph of $f$ is somewhere dense in $\mathbb{R}^{2}$. Therefore there is an open rectangle $U=(a-\delta, b+\delta) \times(c, d) \subset \bar{f}$ where $a<b$ and $\delta>0$. Let $0<r \leq 1$ and we suppose $r \leq d-c$. Pick a point $(a, p) \in \bar{U}$ such that $c+\frac{r}{4}<p<d-\frac{r}{4} . L_{1}$ is the line segment joining the points $(a, p)$ and $\left(b, p-\frac{r}{4}\right)$, and $L_{2}$ is the line segment joining the points $\left(a, p+\frac{r}{8}\right)$ and $\left(b, p+\frac{r}{4}\right) \cdot V$ denotes the trapezoidal region in $U$ that lies 
between $L_{1}$ and $L_{2}$. A function $g: \mathbb{R} \rightarrow \mathbb{R}$ can be obtained from $f$ by shifting all points of $f \cap V$ vertically up to $L_{2}$. Then $d(f, g) \leq \frac{r}{2}$ and $g \notin D \supset A C \cap P R$. To see $g \in W C I V P$, suppose $x<y$ and $g(x) \neq g(y)$. Then since $f$ is dense in $U \backslash \bar{V}$, there is a Cantor set $C \subset(x, y)$ such that $f(C)=g(C)$ lies between $g(x)$ and $g(y)$. In $W C I V P, B\left(g, \frac{r}{16}\right) \subset B(f, r) \backslash D \subset B(f, r) \backslash(A C \cap P R)$. Since $\gamma(f, r, A C \cap P R) \geq \frac{r}{16}, p(f, A C \cap P R) \geq \frac{1}{16}$ implies $A C \cap P R$ is porous at $f$.

Theorem 2. $A C \cap P R$ is porous in $P R$.

Proof. Suppose $f \in \overline{A C \cap P R}$. (This closure taken in PR is the same as the closure taken in $\mathbb{R}^{\mathbb{R}}$.)

Case 1: $\mathrm{f}$ has a point of continuity $x_{0}$.

According to [6], the characteristic function $h:[0,1] \rightarrow[0,1]$ for $C^{0}$ belongs to $P R \backslash D \subset P R \backslash A C$, where $C^{0}$ is the set of nonendpoints of the Cantor ternary set C. Given $0<r \leq 1$, let $g: \mathbb{R} \rightarrow \mathbb{R}$ be the function obtained from $\mathrm{f}$ by gluing a copy of $\mathrm{h}$ at $x_{0}$. Then $g \in P R \backslash A C$ and $d(f, g)<\frac{r}{2}$. Suppose $\phi \in P R$ and $d(\phi, g)<\frac{r}{4}$. Since $\phi$ misses the horizontal line segment $\left[x_{0}-\frac{\delta}{2}, x_{0}+\frac{\delta}{2}\right] \times\left\{f\left(x_{0}\right)\right\}, \phi \notin D$ and so $\phi \notin A C$. Therefore $B\left(g, \frac{r}{4}\right) \subset$ $B(f, r) \backslash A C$.

Case 2: $f$ has no point of continuity.

There is an open rectangle $U=(a, b) \times(c, d) \subset \bar{f}$. Let $0<r \leq \min \{1, d-c\}$. $\mathrm{V}$ is the open rectangle in $\mathrm{U}$ with the same width and center as $\mathrm{U}$ but with height $\frac{r}{2}$. We may suppose $f(a), f(b) \in \bar{U} \backslash \bar{V}$. We obtain a function $g: \mathbb{R} \rightarrow \mathbb{R}$ by moving all points of $f \cap V$ vertically to points of the top horizontal side $\mathrm{H}$ of $\mathrm{V}$ and letting $g=f$ elsewhere. Then $g \in P R \backslash A C$ because $\mathrm{f}$ is dense in $U$ $\backslash V, f \in P R$, and $g \notin D$. By construction, $d(f, g) \leq \frac{r}{2}$ and in the space PR, $B\left(g, \frac{r}{4}\right) \subset B(f, r) \backslash D \subset B(f, r) \backslash A C$.

Both cases show $A C \cap P R$ is porous at $f$.

Theorem 3. If the graph of $f: \mathbb{R} \rightarrow \mathbb{R}$ is dense in $\mathbb{R}^{2}$ and $f \in P R$, then $f \in \overline{A C} \cap \overline{C I V P}$.

Proof. We first show $f \in \bar{D}$. Let $\mathcal{U}$ denote the class of all functions $f: \mathbb{R} \rightarrow \mathbb{R}$ such that for every interval $J \subset \mathbb{R}$ and every set A of cardinality less than $\mathfrak{c}, f(J \backslash A)$ is dense in $\left[\inf _{x \in J} f(x), \sup _{x \in J} f(x)\right]$. According to $[8], \mathcal{U}=\bar{D}$. 
Let $J$ be an interval and suppose $\inf _{x \in J} f(x)<y<\sup _{x \in J} f(x)$. Since $f$ is dense in $\mathbb{R}^{2}$, for each set $\mathrm{O}_{n}=J \times\left(y-\frac{1}{n}, y+\frac{1}{n}\right)$ there exists $x_{n} \in J$ such that $\left(x_{n}, f\left(x_{n}\right)\right) \in O_{n}$. Because $f \in P R$, there exists a perfect set $P_{n}$ in $J$ containing $x_{n}$ such that $f \uparrow P_{n} \subset O_{n}$. For every set $A$ of cardinality less than $\mathfrak{c}, y \in \operatorname{cl}\left(f\left(\cup_{n=1}^{\infty} P_{n} \backslash A\right)\right) \subset \operatorname{cl}(f(J \backslash A))$. Therefore $f \in \mathcal{U}=\bar{D}$. Then $f \in \overline{C I V P}$ because $\overline{C I V P}=P R \cap \bar{D}[2]$. According to [7], since $f \in \bar{D}$ is dense in $\mathbb{R}^{2}$, Conn is dense in each open ball in $\bar{D}$ of radius $\leq 1$ centered at $f$. Therefore $f \in \overline{\text { Conn }}$. By [13], since $f \in \overline{\text { Conn }}$ is dense in $\overline{\mathbb{R}^{2}}, A C$ is dense in each open ball in $\overline{C o n n}$ of radius $\leq 1$ with center $f$. So $f \in \overline{A C}$.

Theorem 4. Ext is a boundary set in SCIVP.

Proof. Suppose $f \in \overline{E x t}$. (This closure is taken in SCIVP.)

Case 1: $f$ is continuous at some point $x_{0}$.

According to [11],the following type example $h:[0,1] \rightarrow[0,1]$ belongs to Conn $\backslash A C$, and the graph of each function within a vertical distance .05 from $h$ is not in $A C$. That is, $B(h, .05) \cap A C=\emptyset$. Let $C$ be the Cantor ternary set in $[0,1],\left\{\left(a_{n}, b_{n}\right): n=1,2,3, \ldots\right\}$ the set of components of $[0,1] \backslash C$, and

$$
h(x)= \begin{cases}\frac{x-a_{n}}{b_{n}-a_{n}} & \text { if } x \in\left[a_{n}, b_{n}\right] \\ \frac{1}{2} & \text { otherwise. }\end{cases}
$$

It turns out $h \in S C I V P$. For each $0<r \leq 1$, the function $g: \mathbb{R} \rightarrow \mathbb{R}$ obtained from $f$ by gluing a copy of $h$ at $x_{0}$ belongs to $S C I V P \backslash A C \subset S C I V P \backslash E x t$, $d(f, g)<\frac{r}{2}$, and $B(g, .025 r) \subset B(f, r) \backslash E x t$. It follows that Ext is porous at $f$.

Case 2: $f$ is discontinuous at every point.

Since the graph of $f$ is somewhere dense in $\mathbb{R}^{2}, \bar{f}$ contains some upright open rectangleU of height $t$. We may suppose $0<r \leq t$, and pick $a<b$ so that $b-a<r$ and the horizontal lines $H_{a}=\mathbb{R} \times\{a\}$ and $H_{b}=\mathbb{R} \times\{b\}$ meet $U$. Move all points of $f$ lying on $H_{a} \cap U$ vertically to points on $H_{b} \cap U$ and leave the remaining points of $f$ alone in order to obtain a function $g: \mathbb{R} \rightarrow \mathbb{R}$ such that $g \notin D$ (because $\left.g \cap H_{a} \cap U=\emptyset\right)$ and $d(f, g)<r$. Also $g \in S C I V P$, really. For, suppose $x<y, g(x) \neq g(y)$, and $K$ is a Cantor set between $g(x)$ and $g(y)$. Let $K_{0}$ be a Cantor set in $K \backslash\{a\}$. Since $f$ is dense in $U$ and $f \in S C I V P$, there is a Cantor set $C \subset(x, y)$ such that $f(C) \subset K_{0}, f \uparrow C$ is continuous, and $g\lceil C=f\lceil C$, which implies $g \in S C I V P$. Therefore $g \in S C I V P \backslash E x t$.

Both cases show that Ext is a boundary set in SCIVP. 
The next theorem follows immediately from a result in [4] that both $S C I V P$ and $C I V P \backslash S C I V P$ are dense in $\overline{C I V P}$.

Theorem 5. SCIVP is not porous in CIVP, but SCIVP is a boundary set in $C I V P$.

Theorem 6. CIVP is porous in WCIVP.

Proof. Let $f \in \overline{C I V P}$.

Case 1: Some $\mathrm{x}_{0}$ is a point of continuity of $\mathrm{f}$.

The same function $h$ and argument given for Case 1 of Theorem 1 can be used here to show that $C I V P$ is porous at $f$ because $h \in W C I V P \backslash P R \subset$ $W C I V P \backslash C I V P$.

Case 2: $f$ has only points of discontinuity.

Since $\overline{C I V P}=W C I V P \cap \bar{D}[2], f \in \bar{D}$ and so by [12] there is an open rectangle $U=(a-\delta, b+\delta) \times(c, d) \subset \bar{f}$ where $a<b$ and $\delta>0$. If $0<r \leq 1$, $r \leq d-c$, and $V=[a, b] \times\left(\frac{c+d}{2}-\frac{r}{4}, \frac{c+d}{2}+\frac{r}{4}\right] \subset U$, then $g: \mathbb{R} \rightarrow \mathbb{R}$ is obtained from the graph of $f$ by shifting all the points of $f \cap V$ vertically up to the horizontal segment $H=[a, b] \times\left\{\frac{c+d}{2}+\frac{3 r}{8}\right\}$ and letting $g=f$ elsewhere. $H$ lies in $U$ but above $V$. Then $g \in W C I V P \backslash C I V P, d(f, g) \leq \frac{5 r}{8}$, and $B\left(g, \frac{r}{5}\right) \subset B(f, r) \backslash C I V P$. It follows that $C I V P$ is porous at $f$.

Theorem 7. If $f \in \overline{W C I V P}$ and the graph of $f$ is dense in $\mathbb{R}^{2}$, then $f \in$ $\overline{C I V P}$.

Proof. We show $f \in W C I V P$ and $f \in \bar{D}$ and then use the fact that $\overline{C I V P}$ $=W C I V P \cap \bar{D}$ according to [2]. Since $f \in \overline{W C I V P}, f$ is the uniform limit of a sequence of functions $f_{n} \in W C I V P$. Suppose $x<y$ and we may suppose $f(x)<f(y)$. Because $f$ is dense in $\mathbb{R}^{2}$, if $w \in(f(x), f(y))$ and $V$ is an open neighborhood of $\mathrm{w}$ in $(f(x), f(y))$, there exist numbers $x_{1}<y_{1}$ in $(x, y)$ such that $w \in\left(f\left(x_{1}\right), f\left(y_{1}\right)\right) \subset V$. For $\varepsilon=\frac{1}{2} \min \left\{f\left(x_{1}\right)-f(x), f(y)-f\left(y_{1}\right)\right\}$, there exists a positive integer $n$ such that for all $z \in \mathbb{R},\left|f(z)-f_{n}(z)\right|<\varepsilon$. There is a Cantor set $C_{1} \subset\left(x_{1}, y_{1}\right)$ such that $f_{n}\left(C_{1}\right) \subset\left(f_{n}\left(x_{1}\right), f_{n}\left(y_{1}\right)\right)$. Then $C_{1} \subset(x, y)$ and $f\left(C_{1}\right) \subset(f(x), f(y))$. Therefore $f \in W C I V P$. There is a Cantor set $C_{2} \subset\left(x_{1}, y_{1}\right)$ such that $f\left(C_{2}\right) \subset\left(f\left(x_{1}\right), f\left(y_{1}\right)\right)$. Therefore since $\mathfrak{c}$-many points of $[x, y]$ map into $V, f \in \mathcal{U}=\bar{D}$.

Remark 1. If the graph of $f: \mathbb{R} \rightarrow \mathbb{R}$ is dense in $\mathbb{R}^{2}$, then $f \in W C I V P$ if and only if $f \in P R$. 
Theorem 8. CIVP is porous in PR.

Proof. Suppose $f \in \overline{C I V P}$.

Case 1: There is a point of continuity $x_{0}$ of $f$.

The characteristic function for the set of nonendpoints of the Cantor ternary set, which is the same function $h \in P R \backslash D$ given for Case 1 of Theorem 2, can be used again here because $h \notin C I V P$. If $0<r \leq 1$ and $g: \mathbb{R} \rightarrow \mathbb{R}$ is the function obtained from $f$ by gluing a copy of $h$ at $x_{0}$, then $g \in P R \backslash C I V P$, $d(f, g)<\frac{r}{2}$, and $B\left(g, \frac{r}{5}\right) \subset B(f, r) \backslash C I V P$.

Case 2: $f$ is discontinuous everywhere.

Let $U=(a, b) \times(c, d) \subset \bar{f}$. We can use the same construction given in Theorem 2 to obtain a function $g \in P R \backslash C I V P$ such that $d(f, g) \leq \frac{r}{2}$. Namely, if $0<r \leq 1, r \leq d-c, V=(a, b) \times\left(\frac{c+d}{2}-\frac{r}{4}, \frac{c+d}{2}+\frac{r}{4}\right) \subset U$, and $f(a), f(b) \in \bar{U} \backslash \bar{V}$, then $\mathrm{g}$ is obtained from the graph of $f$ by shifting all points of $f \cap V$ vertically up to the top horizontal side $H$ of $V$ and letting $g=f$ elsewhere. This time, in the space $P R, B\left(g, \frac{r}{8}\right) \subset B(f, r) \backslash C I V P$ because if $\phi \in B\left(g, \frac{r}{8}\right)$, then there exist points $x<y$ in $(a, b)$ such that $\phi(x)<\frac{c+d}{2}-$ $\frac{r}{8}<\frac{c+d}{2}+\frac{r}{8}<\phi(y)$. If $K$ is a Cantor set in $\left(\frac{c+d}{2}-\frac{r}{8}, \frac{c+d}{2}+\frac{r}{8}\right)$, then there is no Cantor set $C \subset(x, y)$ such that $\phi(C) \subset K$ since $(a, b) \cap$ $\phi^{-1}\left(\frac{c+d}{2}-\frac{r}{8}, \frac{c+d}{2}+\frac{r}{8}\right)=\emptyset$.

Both cases show $C I V P$ is porous in $P R$.

Theorem 9. (a) $P C$ is porous in $Z \omega$ and (b) $P R$ is porous in $Z c$.

Proof. For part $(a)$, let $f \in \overline{P C}=P C[1]$, and for part $(b)$, let $f \in \overline{P R}=$ $P R$ [1].

Case 1: $f$ has some point of continuity $x_{0}$.

The function $h:[0,1] \rightarrow[0,1]$ defined by

$$
h(x)= \begin{cases}\chi_{C^{o}}(x) & \text { if } x \in[0,1] \backslash\left\{\frac{1}{4}\right\} \\ \frac{1}{2} & \text { if } x=\frac{1}{4},\end{cases}
$$

where $C^{0}$ is the set of nonendpoints of the Cantor ternary set, belongs to $Z c \backslash P C \subset(Z \omega \backslash P C) \cap(Z c \backslash P R)[6]$. For each $0<r \leq 1$, the function 
$g: \mathbb{R} \rightarrow \mathbb{R}$ obtained from $f$ by gluing a copy of $h$ at $x_{0}$ belongs to $Z \omega \backslash P C$ for part $(a)$ and belongs to $Z c \backslash P R$ for part $(b)$ and $d(f, g)<\frac{r}{2}$. By construction, (a) $P C$ will be porous at $f$ in $Z \omega$ and (b) $P R$ will be porous at $f$ in $Z c$.

Case 2: $f$ has only discontinuity points.

Since $f$ might not belong to $\bar{D}$, we cannot conclude in general that the graph of $f$ is somewhere dense in $\mathbb{R}^{2}$, but we can in the following first subcase.

Subcase (i): Suppose for every $x \in \mathbb{R}, \bar{f} \cap(\{x\} \times \mathbb{R})$ contains a nondegenerate interval.

For each integer $k$ and positive integer $n$, let $Q_{n k}=\{x \in \mathbb{R}:$ some component of $\bar{f} \cap(\{x\} \times \mathbb{R})$ meets both $\mathbb{R} \times\left\{\frac{k}{n}\right\}$ and $\left.\mathbb{R} \times\left\{\frac{k+1}{n}\right\}\right\}$. Each $Q_{n k}$ is closed and $\mathbb{R}=\cup_{n, k} Q_{n k}$. By the Baire Category Theorem, some $Q_{n k}$ is somewhere dense in $\mathbb{R}$ and therefore contains an interval $(a, b)$. This shows $U=(a, b) \times\left(\frac{k}{n}, \frac{k+1}{n}\right) \subset \bar{f}$. Let $0<r \leq \frac{1}{n}$. Choose a point $(c, f(c)) \in U$ such that the distance from $f(c)$ to each of $\frac{k}{n}$ and $\frac{k+1}{n}$ is $>\frac{r}{4}$, and let $V=(a, b) \times\left(f(c)-\frac{r}{4}, f(c)+\frac{r}{4}\right)$. A function $g: \mathbb{R} \rightarrow \mathbb{R}$ for which $d(f, g) \leq \frac{r}{2}$ can be obtained from $f$ by moving all points of $f \cap V$ except for $(c, f(c))$ vertically to $(a, b) \times\left\{f(c)+\frac{r}{4}\right\}$. Then for part $(a), g \in Z \omega \backslash P C$ (because $f$ is dense in $U), B\left(g, \frac{r}{8}\right) \subset B(f, r) \backslash P C$, and so $P C$ is porous at $f$ in $Z \omega$. For part $(b), g \in Z c \backslash P C, B\left(g, \frac{r}{8}\right) \subset B(f, r) \backslash P R$, and so $P R$ is porous at $f$ in $Z c$.

Subcase (ii): Suppose there exists an $x_{0} \in \mathbb{R}$ such that the closed set $\bar{f} \cap$ $\left(\left\{x_{0}\right\} \times \mathbb{R}\right)$ is totally disconnected.

Let $0<r \leq 1$, and choose a point $\left(x_{0}, a\right) \notin \bar{f} \cap\left(\left\{x_{0}\right\} \times \mathbb{R}\right)$ such that $\frac{r}{4}<a-f\left(x_{0}\right)<\frac{r}{2}$. For part $(a)$, since $f \in P C$, the graph of $f$ is bilaterally dense in itself, and so there exist disjoint countable dense subsets $A_{1}$ and $A_{2}$ of the graph of $f$. Then $A_{1}$ and $A_{2}$ are each bilaterally dense in itself and bilaterally dense in the graph of $f$. For part $(b)$, since $f \in P R$, there exist disjoint bilaterally c-dense subsets $A_{1}$ and $A_{2}$ of the graph of $f$. For both parts $(a)$ and $(b)$, define $g: \mathbb{R} \rightarrow \mathbb{R}$ for which $d(f, g) \leq \frac{3 r}{4}$ by 


$$
g(x)= \begin{cases}a+\frac{r}{4} & \text { if }(x, f(x)) \in A_{1} \cap\left(\mathbb{R} \times\left(a-\frac{r}{2}, a+\frac{r}{2}\right)\right) \text { and } x \neq x_{0} \\ a-\frac{r}{4} & \text { if }(x, f(x)) \in\left(f \backslash A_{1}\right) \cap\left(\mathbb{R} \times\left(a-\frac{r}{4}, a+\frac{r}{4}\right)\right) \\ a & \text { if } x=x_{0} \\ f(x) & \text { otherwise. }\end{cases}
$$

Then in part ( $a), g \in Z \omega \backslash P C$ because each $A_{i}$ is dense in the graph of $f$ and $\left(x_{0}, a\right)$ is an isolated point of the graph of $g$. Since $B\left(g, \frac{r}{8}\right) \subset B(f, r) \backslash P C$, $P C$ is porous at $f$ in $Z \omega$. In part $(b), g \in Z c \backslash P R$ and since $B\left(g, \frac{r}{8}\right) \subset$ $B(f, r) \backslash P R, P R$ is porous at $f$ in $Z c$.

Theorem 10. $P R$ is porous in $P C$.

Proof. Suppose $f \in \overline{P R}=P R[1]$.

Case 1: $f$ is continuous at some $x_{0}$.

If $h:[0,1] \rightarrow[0,1]$ is the characteristic function of the irrationals in $[0,1]$, then $h \in P C \backslash P R[6]$. For every $0<r \leq 1$, glue a copy of $h$ at $x_{0}$ in order to obtain from $f$ a function $g \in P C \backslash P R$ such that $d(f, g)<\frac{r}{2}$ and to see that $P R$ is porous at $f$.

Case 2: $f$ is continuous nowhere.

Subcase (i): For every $x \in \mathbb{R}, \bar{f} \cap(\{x\} \times \mathbb{R})$ contains a nondegenerate interval.

Since the graph of $f$ is somewhere dense in $\mathbb{R}^{2}$, there is an open rectangle $U=(a, b) \times(c, d) \subset \bar{f}$. Let $0<r \leq 1, r \leq d-c$, and pick a point $(p, f(p)) \in U$ such that the rectangle $V=(a, b) \times\left(f(p)-\frac{r}{4}, f(p)+\frac{r}{4}\right) \subset$ $(a, b) \times\left[f(p)-\frac{r}{4}, f(p)+\frac{r}{4}\right] \subset U$. Let $A$ be a countable dense subset of $f \cap V$. A function $g: \mathbb{R} \rightarrow \mathbb{R}$ can be obtained from $f$ by moving all points of $f \cap V$ except for $\{(p, f(p))\} \cup A$ vertically to $(a, b) \times\left\{f(p)+\frac{r}{4}\right\}$. Then $g \in P C \backslash P R$ (since $A$ is dense in $f \cap V$ and $f$ is dense in $U$ ), $d(f, g) \leq \frac{r}{2}$, and $B\left(g, \frac{r}{8}\right) \subset B(f, r) \backslash P R$. So $P R$ is porous at $f$.

Subcase (ii): There is an $x_{0} \in \mathbb{R}$ such that $\bar{f} \cap\left(\left\{x_{0}\right\} \times \mathbb{R}\right)$ is totally disconnected.

For each $0<r \leq 1$, there is a point $\left(x_{0}, a\right) \notin \bar{f}$ such that $\frac{r}{4}<a-f\left(x_{0}\right)<$ $\frac{r}{2}$. Let $B_{1}$ and $B_{2}$ be disjoint countable subsets of $f$. Then $B_{1}$ and $B_{2}$ are 
each bilaterally dense in itself and bilaterally dense in the graph of $f$. Define $g: \mathbb{R} \rightarrow \mathbb{R}$ by

$g(x)= \begin{cases}a+\frac{r}{4} & \text { if }(x, f(x)) \in\left(f \backslash B_{1}\right) \cap\left(\mathbb{R} \times\left(a-\frac{r}{4}, a+\frac{r}{4}\right)\right) \\ a & \text { if } x=x_{0} \text { or } \\ & (x, f(x)) \in B_{2} \cap\left[\mathbb{R} \times\left(\left(a-\frac{r}{2}, a-\frac{r}{4}\right) \cup\left(a+\frac{r}{4}, a+\frac{r}{2}\right)\right)\right] \\ f(x) \quad \text { elsewhere. }\end{cases}$

Then $g \in P C \backslash P R, d(f, g) \leq \frac{r}{2}$, and $B\left(g, \frac{r}{8}\right) \subset B(f, r) \backslash P R$. Therefore $P R$ is porous at $f$.

Theorem 11. $P R$ is porous in $P B$.

Proof. Let $f \in \overline{P R}=P R$ and let $x_{0} \in \mathbb{R}$. Since $f$ has a perfect road at $x_{0}$, there exists a perfect set $P_{0}$ having $x_{0}$ as a bilateral limit point such that $f \nmid P_{0}$ is continuous at $x_{0}$. Let $0<r \leq 1$ and $S=\mathbb{R} \times\left(f\left(x_{0}\right)-\frac{r}{4}, f\left(x_{0}\right)+\frac{r}{4}\right)$. Define $g(x)=f\left(x_{0}\right)+\frac{r}{4}$ whenever

(1) $(x, f(x)) \in S$ and $x \neq x_{0}$ or

(2) $(x, f(x)) \in \mathbb{R} \times\left\{f\left(x_{0}\right)-\frac{r}{4}\right\}$ and there exists a perfect road $P$ at $x$ such that either of $f \uparrow(P \cap(-\infty, x))$ or $f \uparrow(P \cap(x, \infty))$ is contained in $\bar{S}$, and $(x, f(x))$ is not a limit point of $f \backslash \bar{S}$.

Both $P \cap(-\infty, x]$ and $P \cap[x, \infty)$ are perfect sets containing x. Define $g(x)=f(x)$ for all other $x$. Suppose $I$ and $J$ are open intervals with $I \cap$ $g^{-1}(J)$ uncountable. We may suppose $J$ contains $f\left(x_{0}\right)-\frac{r}{4}$ or $f\left(x_{0}\right)+\frac{r}{4}$. Let $w \in I \cap g^{-1}(J)$. Therefore $g(w) \in J$. If $g(w)=f(w)$, then there exists a perfect set $P_{1}$ containing w such that $g \uparrow P_{1}$ is continuous at $w$, and so $I \cap g^{-1}(J)$ contains a perfect subset of $P_{1}$. If $g(w) \neq f(w)$, then $g(w)=$ $f\left(x_{0}\right)+\frac{r}{4}$ and either by $(1),(w, f(w)) \in S$ (in which case $f$ has a perfect road $P_{2}$ at $\left.w\right)$ or by $(2), f(w)=f\left(x_{0}\right)-\frac{r}{4}$ and there exists a perfect set $P_{3}$ containing $w$ such that $f \uparrow\left(P_{3} \backslash\{w\}\right) \subset S$. So $I \cap g^{-1}(J)$ contains a perfect subset of either $P_{2}$ or $P_{3}$. Therefore $g \in P B \backslash P R$ and $d(f, g) \leq \frac{r}{2}$. In $P B$, the open ball $B\left(g, \frac{r}{8}\right) \subset B(f, r) \backslash P R$. Since $p(f, P R) \geq \frac{1}{8}, P R$ is porous at $f$.

Theorem 12. $\overline{P B}=P B$. 
Proof. Suppose $f \in \overline{P B}$ and $I$ and $J$ are open intervals such that $I \cap$ $f^{-1}(J)$ is uncountable. Then $f$ is the uniform limit of a sequence of functions $f_{n} \in P B$. There exist a positive number $\varepsilon \leq 1$, a positive integer $N$, and an interval $(c, d) \subset(c-\varepsilon, d+\varepsilon) \subset J$ such that $I \cap f^{-1}(c, d)$ is uncountable and $\left|f(x)-f_{n}(x)\right|<\frac{\varepsilon}{2}$ for all $n \geq N$ and for all $x \in \mathbb{R}$. Therefore for all $n \geq N, I \cap f^{-1}(c, d) \subset I \cap f_{n}^{-1}\left(c-\frac{\varepsilon}{2}, d+\frac{\varepsilon}{2}\right)$, which since uncountable contains a perfect set $P_{n}$. Because $f\left(P_{n}\right) \subset f\left(I \cap f_{n}^{-1}\left(c-\frac{\varepsilon}{2}, d+\frac{\varepsilon}{2}\right)\right) \subset J$, $P_{n} \subset I \cap f^{-1}(J)$. Therefore $f \in P B$.

The following result is different from all the others.

Theorem 13. $Z c$ is not a boundary set in $Z \omega$.

Proof. We must show that $Z c$ contains an open ball of $Z \omega$. Let $\mathbb{Q}$ denote the set of rational numbers and $\left\{F_{q}: q \in \mathbb{Q}\right\}$ denote a collection of pairwise disjoint $\mathfrak{c}$-dense subsets of $\mathbb{R}$. Like in $[9]$, define $f=\sum_{q \in \mathbb{Q}} q \chi_{F_{q}}$. Then $f \in$ $Z c$. Let $0<\varepsilon \leq 1$ and suppose $g \in Z \omega$ with $d(f, g)<\varepsilon$. Let $a \in \mathbb{R}$ and choose a rational number $q>a+\varepsilon$. If $x \in F_{q}$, then $f(x)=q$, and since $|q-g(x)|=|f(x)-g(x)| \leq d(f, g)<\varepsilon \leq 1$, then $g(x)>a$. Therefore $F_{q} \subset\{x: g(x)>a\}$, which implies $\{x: g(x)>a\}$ is $\mathfrak{c}$-dense in $\mathbb{R}$ and hence bilaterally $\mathfrak{c}$-dense in itself. Similarly, $\{x: g(x)<a\}$ is bilaterally $\mathfrak{c}$-dense in itself. Then $g \in Z c$. This shows $B(f, \varepsilon) \subset Z c$. So $Z c$ is not a boundary set in $Z \omega$ (and consequently not porous in $Z \omega$ ).

\section{References}

[1] K. Banaszewski, Algebraic properties of $\varepsilon$-continuous functions, Real Anal. Exchange, 18 (1992-93), 153-168.

[2] K. Banaszewski, Algebraic properties of functions with the Cantor intermediate value property, Math. Slovaka, 48 (1998), to appear.

[3] K. Banaszewski, Algebraic properties of functions with the Cantor intermediate value property. Abstract, Real Anal. Exchange, 21(1995-96), $33-35$.

[4] K. Banaszewski and T. Natkaniec, Sierpinski-Zygmund functions that have the Cantor intermediate value property, Real Anal. Exchange, 24 (1998-99), 827-835.

[5] J. B. Brown, Nowhere dense Darboux graphs, Colloq. Math., 20 (1969), 243-247. 
[6] J. B. Brown, P. Humke, and M. Laczkovich, Measurable Darboux functions, Proc. Amer. Math. Soc., 102 (1988), 603-610.

[7] A. M. Bruckner and J. G. Ceder, On jumping functions by connected sets, Czech. Math. J., 22 (1972), 435-448.

[8] A. M. Bruckner, J. G. Ceder, M. Weiss, Uniform limits of Darboux functions, Colloq. Math., 15 (1966), 65-77.

[9] K. Ciesielski and J. Jastrzebski, Darboux-like functions within the classes of Baire one, Baire two, and additive functions, Topology Appl., 103(2) (2000), 203-219.

[10] R. G. Gibson and T. Natkaniec, Darboux like functions, Real Anal. Exchange, 22 (1996-97), 492-533.

[11] J. Jastrzebski, An answer to a question of R. G. Gibson and F. Roush, Real Anal. Exchange, 15 (1989-90), 340-341.

[12] F. B. Jones and E. S. Thomas, Jr., Connected $G_{\delta}$ graphs, Duke Math. J. 33 (1966), 341-345.

[13] H. Rosen, Porosity in spaces of Darboux-like functions, Real Anal. Exchange, 26 (2000-01), 195-200. 
HARVEy Rosen 